\title{
On the Monomial Birational Maps of the Projective Space
}

\author{
GÉRARD GONZALEZ-SPRINBERG ${ }^{1}$ and IVAN PAN ${ }^{2}$ \\ ${ }^{1}$ Institut Fourier, Université de Grenoble I UMR 5582 CNRS-UJF 38402 Saint-Martin-d'Hères, France \\ ${ }^{2}$ Instituto de Matemática, Universidade Federal do Rio Grande do Sul, 91501-970 Porto Alegre, Brasil
}

Manuscript received on December 18, 2002; accepted for publication on April 30, 2003;

presented by ARON SIMIs

\begin{abstract}
We describe the group structure of monomial Cremona transformations. It follows that every element of this group is a product of quadratic monomial transformations, and geometric descriptions in terms of fans.
\end{abstract}

Key words: monomial birational maps, toric varieties.

\section{INTRODUCTION}

The best known birational map of $\mathbb{P}^{r}$ is maybe the standard Cremona transformation $S_{r}: \mathbb{P}^{r} \rightarrow-\mathbb{P}^{r}$ defined by $S_{r}=\left(X_{0}^{-1}: \cdots: X_{r}^{-1}\right)$. For $r=2$ there is a geometric description in the classic references on this subject; moreover a Max Noether's famous theorem shows that every birational map of $\mathbb{P}^{2}$ is a composition of automorphisms and $S_{2}$, and then that every Cremona transformation is a composition of quadratic ones. If $r>2$ there is no analogous to Noether's theorem (Hudson 1927, Katz 1992, Pan 1999). In this note we consider birational maps generalizing $S_{r}$. Our approach is based in a toric point of view and the property that $S_{r}$ stabilizes the open set $X_{0} \cdots X_{r} \neq 0$. We consider birational maps of $\mathbb{P}^{r}$ with this property. In the second paragraph of (Russo and Simis 2001) the birationality of these maps is characterized in terms of certain syzygies as an application of a more general criterion; see also (Simis and Villarreal 2002): compare their Proposition 1.1 with our Proposition 3.1.

Mathematics Subject Classification: 14E07, 14M25.

Correspondence to: Ivan Pan

E-mail: pan@mat.ufrgs.br / gonsprin@ujf-grenoble.fr 


\section{MONOMIAL CREMONA TRANSFORMATIONS}

Let $N$ be a rank $r$ free $\mathbb{Z}$-module, $\mathbb{K}$ an algebraically closed field, $\mathbb{K}^{*}:=\mathbb{K} \backslash\{0\}$. Let $\mathbb{T}=\mathbb{T}_{N}:=$ $N \otimes_{\mathbb{Z}} \mathbb{K}^{*}$ be the algebraic torus associated to $N$ over $\mathbb{K}$. The action of $\mathbb{T}$ on itself induces a natural inclusion $\imath$ of the torus in the group Aut $(\mathbb{T})$ of the algebraic variety's automorphisms of $\mathbb{T}$; we consider the subgroup $G_{\mathbb{T}}$ of Aut $(\mathbb{T})$ given by the algebraic group's automorphisms of $\mathbb{T}$. Note that, with an appropriate choice of a basis of $N$, we may identify $\mathbb{T}$ to $\left(\mathbb{K}^{*}\right)^{r}$. Since $\mathbb{K}$ is algebraically closed an automorphism in Aut $(\mathbb{T})$ may be written in the form $F=\left(F_{1}, \ldots, F_{r}\right)$ with

$$
F_{i}=\lambda_{i} x_{1}^{a_{i 1}} \cdots x_{r}^{a_{i r}}
$$

where $\lambda_{i} \in \mathbb{K}^{*}, a_{i j} \in \mathbb{Z}, 1 \leq i, j \leq r$.

Lemma 2.1. There is a split exact sequence of groups

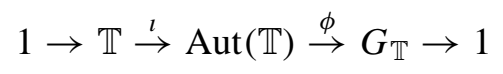

where $\phi(F):=\imath\left(F\left(1_{\mathbb{T}}\right)^{-1}\right) \circ F$, for $F \in \operatorname{Aut}(\mathbb{T})$.

Proof. One has $\phi(F) \in G_{\mathbb{T}}$, because $\lambda_{i}^{-1} F_{i}$ is a character of the torus, $1 \leq i \leq r$; it is easily seen that the sequence is exact. The inclusion of $G_{\mathbb{T}}$ in $\operatorname{Aut}(\mathbb{T})$ is a section of $\phi$, hence the sequence splits.

In the following we consider the projective space $\mathbb{P}^{r}$ over $\mathbb{K}$ as a toric variety, e.g., a compactification of the torus $\mathbb{T}=\mathbb{T}_{N}$ associated to a complete regular fan of $N_{\mathbb{R}}:=N \otimes_{\mathbb{Z}} \mathbb{R}$ with $(r+1)$ cones of dimension 1. (One standard reference for toric varieties is (Oda 1988)).

Definition 2.2. A monomial Cremona transformation is a birational map $F: \mathbb{P}^{r} \rightarrow \mathbb{P}^{r}$ defined on $\mathbb{T}$ and such that $F(\mathbb{T}) \subset \mathbb{T}$.

We note $\operatorname{Bir}_{\mathbb{T}}\left(\mathbb{P}^{r}\right)$ the group of these transformations; there is a natural isomorphism $\operatorname{Bir}_{\mathbb{T}}\left(\mathbb{P}^{r}\right) \stackrel{\sim}{\longrightarrow} \operatorname{Aut}(\mathbb{T})$.

Proposition 2.3. Fixing a basis of the lattice N, there exists an isomorphism

$$
\operatorname{Bir}_{\mathbb{T}}\left(\mathbb{P}^{r}\right) \simeq \mathbb{T} \rtimes \mathrm{GL}_{r}(\mathbb{Z}) .
$$

Proof. It follows from Lemma 2.1. Given $F \in \operatorname{Bir}_{\mathbb{T}}\left(\mathbb{P}^{r}\right)$, we associate $\left(F\left(1_{\mathbb{T}}\right),\left(a_{i j}\right)\right)$, where $\left(a_{i j}\right)$ is the $r \times r$-matrix corresponding to $\phi(F) \in G_{\mathbb{T}}$ via the equality (1) and the isomorphism $G_{\mathbb{T}} \stackrel{\sim}{\longrightarrow} \mathrm{GL}_{r}(\mathbb{Z})$ induced by the choice of the basis of $N$.

REMARK 2.4. The action of $\mathrm{GL}_{r}(\mathbb{Z})$ on $\mathbb{T}$ corresponding to the semidirect product in the proposition is

$$
\left(a_{i j}\right) \cdot\left(\lambda_{1}, \ldots, \lambda_{r}\right)=\left(\prod_{j} \lambda_{j}^{a_{1 j}}, \ldots, \prod_{j} \lambda_{j}^{a_{r j}}\right) .
$$


COROLlaRY 2.5. The center of $\operatorname{Bir}_{\mathbb{T}}\left(\mathbb{P}^{r}\right)$ is trivial. The center of the subgroup $\left\{F: F\left(1_{\mathbb{T}}\right)=1_{\mathbb{T}}\right\}$ is $\left\langle S_{r}\right\rangle$.

As a consequence of Proposition 2.3 we obtain an analogous to the M. Noether's theorem (on the generators of the Cremona group of $\mathbb{P}^{2}$ ) in arbitrary dimension for the monomial case.

THEOREM 2.6. The group $\operatorname{Bir}_{\mathbb{T}}\left(\mathbb{P}^{r}\right)$ is generated by a quadratic transformation and the linear monomial automorphisms. Consequently, every birational map is a product of quadratic transformations.

Proof. It follows from Proposition 2.3 and the fact that $\mathrm{GL}_{r}(\mathbb{Z})$ is generated by a transvection and two permutations (Coxeter and Moser 1957, Trott 1962) which induce respectively a quadratic transformation and two linear maps in $\operatorname{Bir}_{\mathbb{T}}\left(\mathbb{P}^{r}\right)$.

\section{DEGREES AND MATRICES}

Let $X_{0}, \ldots, X_{r}$ be homogeneous coordinates in $\mathbb{P}^{r}, x_{i}:=X_{i} / X_{0}, 1 \leq i \leq r$, the affine coordinates corresponding to the canonical basis of $N=\mathbb{Z}^{r}$. Every non-constant monomial rational map $F: \mathbb{P}^{r} \rightarrow \mathbb{P}^{r}$ satisfying $F\left(1_{\mathbb{T}}\right)=1_{\mathbb{T}}$ may be written uniquely in the form

$$
F=\left(X_{0}^{\alpha_{00}} \cdots X_{r}^{\alpha_{0 r}}: \cdots: X_{0}^{\alpha_{r 0}} \cdots X_{r}^{\alpha_{r r}}\right)
$$

such that:

(i) $\alpha\left(e_{j}\right) \in \partial C_{+}, 0 \leq j \leq r$,

(ii) $\alpha(e)=\operatorname{deg}(F) e$, where $\alpha=\alpha_{F}=\left(\alpha_{i j}\right), e_{j}, 0 \leq j \leq r$, is the canonical basis of $\mathbb{Z}^{r+1}$, $e=\sum_{j} e_{j}, C_{+}$is the cone $\sum_{j} \mathbb{R}_{\geq 0} e_{j}$ and $\partial C_{+}$its boundary. The positive integer $\operatorname{deg}(F)$ is the degree of $F$, e.g. $\operatorname{deg}(F)=\sum_{j} \alpha_{i j}, \forall i$.

The map $F$ is birational if and only if there exists an integer $(r+1) \times(r+1)$ - matrix $\beta$, satisfying (i), (ii), and $v \in \mathbb{Z}^{r+1}$ such that:

(iii) $\alpha \beta=I d+e \cdot{ }^{t} v$.

In the affine open set $X_{0} \cdots X_{r} \neq 0$ one may write $F$ as

$$
F=\left(1: x_{1}^{a_{11}} \cdots x_{r}^{a_{1 r}}: \cdots: x_{1}^{a_{11}} \cdots x_{r}^{a_{r r}}\right) .
$$

The matrix $A:=\left(a_{i j}\right)$ is the matrix of exponents of (1).

Proposition 3.1. Let $F \in \operatorname{Bir}_{\mathbb{T}}\left(\mathbb{P}^{r}\right)$ be such that $F\left(1_{\mathbb{T}}\right)=1_{\mathbb{T}}, \alpha=\alpha_{F}, d=\operatorname{deg}(F)$. There exists a unique isomorphism $\bar{\alpha}$ such that the following diagram is commutative and with exact lines.

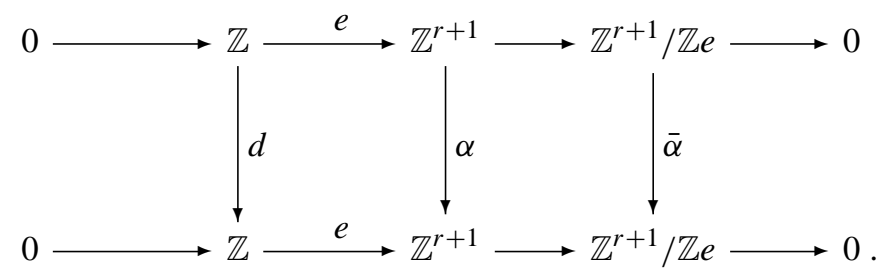


One has $d=|\operatorname{det}(\alpha)|$ and $A$ is the matrix of $\bar{\alpha}$ in the basis $\overline{e_{1}}, \ldots, \overline{e_{r}}$.

Proof. It follows from properties (ii) and (iii) of $\alpha$ and the form in which $\alpha$ is written in the basis $e, e_{1}, \ldots, e_{r}$ because (2) and (3) imply $a_{i j}=\alpha_{i j}-\alpha_{0 j}, 1 \leq i, j \leq r$.

\section{REMARK 3.2.}

(a) $\mathbb{Z}^{r+1} / \alpha\left(\mathbb{Z}^{r+1}\right)$ is cyclic of order $d$.

(b) We obtain $\alpha$ from $A$ as follows:

$$
\begin{aligned}
& \alpha_{0 j}=-\min \left(0, a_{i j}, 1 \leq i \leq r\right), \text { for } 1 \leq j \leq r ; \\
& \alpha_{00}=\max \left(0, \sum_{j} a_{i j}, 1 \leq i \leq r\right) \\
& \alpha_{i j}=a_{i j}+\alpha_{0 j}, \text { for } 1 \leq i, j \leq r \\
& \alpha_{i 0}=\alpha_{00}-\sum_{j} a_{i j}, \text { for } 1 \leq i \leq r .
\end{aligned}
$$

ExAmple 3.3. Let $A$ be the matrix whose lines are $(1,0,0),(a, 1,0),(0, a, 1), a \geq 1$. Then

$$
F_{A}=\left(X_{0}^{a+1}: X_{0}^{a} X_{1}: X_{1}^{a} X_{2}: X_{2}^{a} X_{3}\right)
$$

and

$$
F_{A^{-1}}=\left(X_{0}^{a^{2}-a+1} X_{1}^{a} X_{2}^{a}: X_{0}^{a^{2}-a} X_{1}^{a+1} X_{2}^{a}: X_{0}^{a^{2}} X_{2}^{a+1}: X_{1}^{a^{2}+a} X_{3}\right)
$$

EXAMPLE 3.4. Finite subgroup of $\operatorname{Bir}_{\mathbb{T}}\left(\mathbb{P}^{r}\right)$ are obtained by this method. For example the Weyl's group of type $W\left(D_{r}\right), r \geq 3$, and order $2^{r-1} r$ !, as a Cremona subgroup may be represented as the subgroup generated by an involution of degree 3

$$
F_{\alpha_{r}}=\left(X_{0} X_{r-1} X_{r}: X_{1} X_{r-1} X_{r}: \cdots: X_{r-2} X_{r-1} X_{r}: X_{0}^{2} X_{r-1}: X_{0}^{2} X_{r}\right)
$$

and the linear automorphisms $F_{\alpha_{i}}$ permuting $X_{i}$ with $X_{i+1}, 1 \leq i<r$. In an analogous form one obtains a representation of the group $W\left(B_{r}\right), r \geq 2$, of order $2^{r} r$ !, generated by an involution of degree 2

$$
F_{\beta_{r}}=\left(X_{0} X_{r}: X_{1} X_{r}: \cdots: X_{r-1} X_{r}: X_{0}^{2}\right)
$$

and the preceding $r-1$ permutations. 


\section{GEOMETRIC DESCRIPTION, EXAMPLES}

Let $F \in \operatorname{Bir}_{\mathbb{T}}\left(\mathbb{P}^{r}\right)$; we denote by $B_{F}$ its base-scheme. By composing $F$ with an automorphism induced by an element of the torus we may assume that $F\left(1_{\mathbb{T}}\right)=1_{\mathbb{T}}$. e.g. $F=\left(1_{\mathbb{T}}, A\right) \in$ $\mathbb{T} \rtimes \mathrm{GL}_{r}(\mathbb{Z})$, we note $F_{A}=F$; the base-scheme is not changed.

The union of the fundamental hyperplanes $\mathbb{P}^{r} \backslash \mathbb{T}$ contains the base-points set.

Let $e_{1}, \ldots, e_{r}$ the canonical basis for $N=\mathbb{Z}^{r}, e:=e_{1}+\cdots+e_{r}, \Delta$ the fan associated to the faces of the simplex $\delta=\left[e_{1}, \ldots, e_{r},-e\right]$ and $A(\Delta)$ the same with respect to the simplex $A(\delta)$. We consider $\mathbb{P}^{r}$ defined by $\Delta$; let $\Sigma$ be a fan that is a common subdivision for $\Delta$ and $A(\Delta)$.

Proposition 4.1. One has a commutative diagram

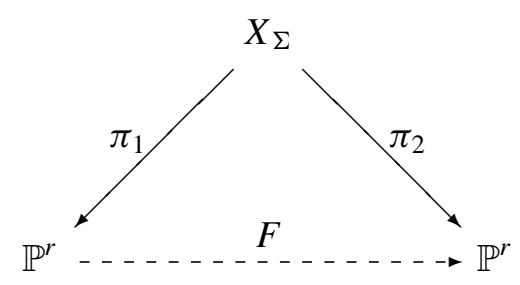

where $X_{\Sigma}$ is the toric variety associated to $\Sigma, \pi_{1}$ is equivariant and $\pi_{2}$ is equivariant relatively to the automorphism of the torus induced by $A$.

Proof. From (3), il follows that $F$ induce $F^{*}: \mathbb{K}[\mathbb{T}] \rightarrow \mathbb{K}[\mathbb{T}]$ which corresponds, by duality, to the lattice's automorphism $A: N \rightarrow N$.

Example 4.2. Let $A=\left(\begin{array}{ll}1 & 0 \\ 1 & 1\end{array}\right)$ be, $F_{A}=\left(X_{0}^{2}, X_{0} X_{1}, X_{1} X_{2}\right)$. One obtains a (regular) fan $\Sigma$ that is a subdivision of $\Delta$ and $A(\Delta)$ by blowing-up the closed orbits associated to the cones $\left\langle e_{1}, e_{2}\right\rangle$ and $\left\langle e_{1},-e\right\rangle$ and then $\left\langle-e_{2},-e\right\rangle$.

Example 4.3. Let $A=-I d$, then $F_{A}=S_{r}$. One obtains a (regular) fan $\Sigma$ by the elementary subdivisions of $\Delta$ (resp. of $-\Delta$ ), successively, of the cones of decreasing dimensions from $r$ to 2 . For example, if $r=3$, let $\Sigma_{0}$ (resp. $\Sigma_{0}^{\prime}$ ) be the fan obtained by the elementary subdivisions of the 4 maximal cones of $\Delta$ (resp. $-\Delta$ ), and $\Sigma_{1}$ (resp. $\Sigma_{1}^{\prime}$ ), and the following subdivisions corresponding to the 6 cones of dimension 2 of $\Delta$ (resp. $-\Delta$ ). One has $\Sigma=\Sigma_{1}=\Sigma_{1}^{\prime}$. The toric variety $V_{\Sigma_{0}}$ (resp. $V_{\Sigma_{0}^{\prime}}$ ) is the blowing-up of $\mathbb{P}^{3}$ in 4 points and $V_{\Sigma_{1}}$ (resp. $V_{\Sigma_{1}^{\prime}}$ ) is the blowing-up of these in the strict transforms of the 6 lines. The induced birational map $V_{\Sigma_{0}} \rightarrow-V_{\Sigma_{0}^{\prime}}$ is composition of 6 flops, e.g., corresponding to small resolutions of singularities of type an affine cone over a smooth quadric, associated to the 6 faces of the convex polyhedron $P_{\Delta}:=\operatorname{Conv}(\Delta \cup(-\Delta))$.

On the other hand, the (normalized) blowing-up of the base-scheme $B$ is the toric variety associated to the fan $\Sigma(B)$ defined by the faces of the polyhedron $P(B)=\operatorname{Conv}\left(P_{\Delta} \cup C\right)$, where $C$ is the set of sums of the vertices of a diagonal of each 2-face of $P_{\Delta}$. The toric variety $V_{\Sigma(B)}$ has 12 singular points of type an affine cone over a smooth quadric. Finally, the fan $\Sigma$ is a regular subdivision of $\Sigma(B)$ and the induced morphism $V_{\Sigma} \rightarrow V_{\Sigma(B)}$ is a minimal resolution of $V_{\Sigma(B)}$. 


\section{ACKNOWLEDGMENTS}

The second author was a visiting Professor at Institut Fourier and also partially supported by the Cooperação Franco-Brasileira.

\section{RESUMO}

Descrevemos a estrutura do grupo das transformações de Cremona monomiais. Concluímos que todo elemento deste grupo é um produto de aplicações monomiais quadráticas e damos descrições geométricas em termos de leques.

Palavras-chave: aplicações birracionais monomiais, variedades tóricas.

\section{REFERENCES}

CoXeter H ANd Moser W. 1957. Generators and relations for Discrete Groups. Springer-Verlag.

Hudson HP. 1927. Cremona Transformations in Plane and Space. Cambridge at the University Press.

Katz S. 1992. Recent works on Cremona Transformations. Research Notes in Math. 2: 109-119, Sundance 90 .

OdA T. 1988. Convex Bodies and Algebraic Geometry. Springer-Verlag.

PAN I. 1999. Une remarque sur la génération du groupe de Cremona. Bol Soc Bras Mat 30: 95-98.

Russo F AND Simis A. 2001. On birational maps and Jacobian matrices. Compositio Math 126: 335-358.

Simis A and Villarreal R. 2002. Constraints for the Normality of Monomial Subrings and birationality, to appear in Proc. of the A.M.S.

Trotт S. 1962. A pair of generators for the unimodular group. Can Math Bull 5: 245-252. 